\title{
An algorithm for the reconstruction of neutrino-induced showers in the ANTARES neutrino telescope
}

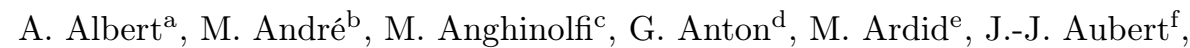

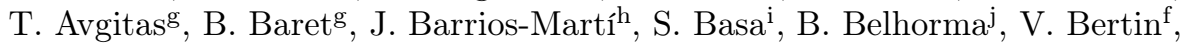
S. Biagi ${ }^{k}$, R. Bormuth ${ }^{1, m}$, S. Bourret ${ }^{g}$, M.C. Bouwhuis ${ }^{1}$, H. Brânzaş ${ }^{n}$,

R. Bruijn ${ }^{1, o}$, J. Brunner ${ }^{\mathrm{f}}$, J. Busto ${ }^{\mathrm{f}}$, A. Capone ${ }^{\mathrm{p}, \mathrm{q}}$, L. Caramete $^{\mathrm{n}}$, J. Carr ${ }^{\mathrm{f}}$,

S. Celli ${ }^{\mathrm{p}, \mathrm{q}, \mathrm{r}}$, R. Cherkaoui El Moursli ${ }^{\mathrm{s}}$, T. Chiarusi ${ }^{\mathrm{t}}$, M. Circella $^{\mathrm{u}}$,

J.A.B. Coelho ${ }^{\mathrm{g}}$, A. Coleirog,h, R. Coniglione ${ }^{\mathrm{k}}$, H. Costantinif ${ }^{\mathrm{f}}$, P. Coyle ${ }^{\mathrm{f}}$,

A. Creusot ${ }^{\mathrm{g}}$, A. F. Díaz ${ }^{\mathrm{v}}$, A. Deschamps ${ }^{\mathrm{w}}$, G. De Bonis ${ }^{\mathrm{p}, \mathrm{q}}$, C. Distefano ${ }^{\mathrm{k}}$,

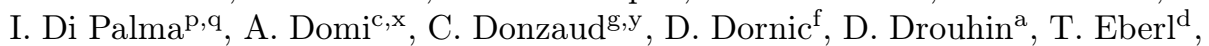

I. El Bojaddaini ${ }^{\mathrm{z}}$, N. El Khayatis ${ }^{\mathrm{s}}$ D. Elsässer ${ }^{\text {aa }}$, A. Enzenhöfer ${ }^{\mathrm{f}}$, A. Ettahiri ${ }^{\mathrm{s}}$,

F. Fassis ${ }^{\mathrm{s}}$, I. Felis ${ }^{\mathrm{e}}$, L.A. Fusco, ${ }^{\mathrm{t}, \mathrm{ab}}$, P. Gay ${ }^{\mathrm{ac}, \mathrm{g}}$, V. Giordano ${ }^{\text {ad }}$, H. Glotin ${ }^{\mathrm{ae}}$,af,

T. Grégoire ${ }^{g}$, R. Gracia Ruiz ${ }^{\mathrm{g}}$, K. Graf ${ }^{\mathrm{d}}$, S. Hallmann ${ }^{\mathrm{d}}$, H. van Haren ${ }^{\text {ag }}$,

A.J. Heijboer ${ }^{1}$, Y. Hellow ${ }^{w}$ J.J. Hernández-Rey ${ }^{h}, J$. Hößl ${ }^{d}$, J. Hofestädt ${ }^{d}$,

C. Hugon ${ }^{\mathrm{c}, \mathrm{x}}$, G. Illuminati ${ }^{\mathrm{h}}$, C.W. James ${ }^{\mathrm{d}}$, M. de Jong ${ }^{\mathrm{l}, \mathrm{m}}$, M. Jongen ${ }^{\mathrm{l}}$,

M. Kadler ${ }^{\text {aa }}$, O. Kalekin ${ }^{\mathrm{d}}$, U. Katz ${ }^{\mathrm{d}}$, D. Kießling ${ }^{\mathrm{d}}$, A. Kouchner ${ }^{\mathrm{g}, \text { af }}$,

M. Kreter ${ }^{\text {aa }}$, I. Kreykenbohm ${ }^{\text {ah }}$, V. Kulikovskiy ${ }^{\text {f,ai }}$, C. Lachaud ${ }^{\mathrm{g}}$,

R. Lahmann ${ }^{\text {d }}$, D. Lefèvre ${ }^{\text {aj }}$, E. Leonora ${ }^{\text {ad,ak }}$, M. Lotze ${ }^{\text {h }}$, S. Loucatos ${ }^{\text {al,g, }}$,

M. Marcelin ${ }^{\mathrm{i}}$, A. Margiotta ${ }^{\mathrm{t}, \mathrm{ab}}$, A. Marinelli ${ }^{\mathrm{am}, \mathrm{an}}$, J.A. Martínez-Mora ${ }^{\mathrm{e}}$,

R. Mele ${ }^{\mathrm{ao}, \mathrm{ap}}$, K. Melis ${ }^{\mathrm{l}, \mathrm{o}}$, T. Michael ${ }^{\mathrm{l}}$, P. Migliozzi ${ }^{\mathrm{ao}}$, A. Moussa $^{\mathrm{z}}, \mathrm{S}_{\text {. Navas }}{ }^{\mathrm{aq}}$,

E. Nezri ${ }^{i}$, M. Organokovir ${ }^{\text {ar }}$ G.E. Păvălaş ${ }^{\mathrm{n}}$, C. Pellegrino ${ }^{\mathrm{t}, \mathrm{ab}}$, C. Perrina ${ }^{\mathrm{p}, \mathrm{q}}$,

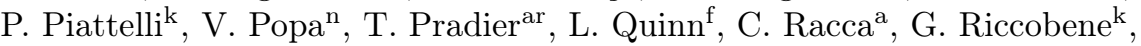

A. Sánchez-Losa ${ }^{u}$, M. Saldaña ${ }^{\mathrm{e}}$, I. Salvadori ${ }^{\mathrm{f}}$, D. F. E. Samtleben ${ }^{\mathrm{l}, \mathrm{m}}$,

M. Sanguineti ${ }^{c, x}$, P. Sapienza ${ }^{k}$, F. Schüssler ${ }^{\text {al }}$, C. Sieger ${ }^{d}$, M. Spurio ${ }^{t, a b}$,

Th. Stolarczyk ${ }^{a l}$, M. Taiuti ${ }^{c, x}$, Y. Tayalatis, A. Trovato ${ }^{k}$, D. Turpin ${ }^{\mathrm{f}}$,

C. Tönnis ${ }^{\mathrm{h}}$, B. Vallage ${ }^{\mathrm{al}, \mathrm{g}}, \mathrm{V}$. Van Elewyck ${ }^{\mathrm{g}, \mathrm{af}}$, F. Versari ${ }^{\mathrm{t}, \mathrm{ab}}$, D. Vivolo ${ }^{\mathrm{ao}, \mathrm{ap}}$,

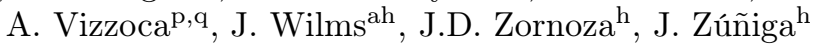

\footnotetext{
${ }^{a}$ GRPHE - Université de Haute Alsace - Institut universitaire de technologie de Colmar, 34 rue du Grillenbreit BP 50568 - 68008 Colmar, France

${ }^{b}$ Technical University of Catalonia, Laboratory of Applied Bioacoustics, Rambla Exposició, 08800 Vilanova i la Geltrú, Barcelona, Spain ${ }^{c}$ INFN - Sezione di Genova, Via Dodecaneso 33, 16146 Genova, Italy

${ }^{d}$ Friedrich-Alexander-Universität Erlangen-Nürnberg, Erlangen Centre for Astroparticle
Fing Physics, Erwin-Rommel-Str. 1, 91058 Erlangen, Germany

e Institut d'Investigació per a la Gestió Integrada de les Zones Costaneres (IGIC) - Universitat

Politècnica de València. C/ Paranimf 1, 46730 Gandia, Spain

${ }^{f}$ Aix Marseille Univ, CNRS/IN2P3, CPPM, Marseille, France

${ }^{g}$ APC, Univ Paris Diderot, CNRS/IN2P3, CEA/Irfu, Obs de Paris, Sorbonne Paris Cité, France

${ }^{h}$ IFIC - Instituto de Física Corpuscular (CSIC - Universitat de València) c/ Catedrático José Beltrán, 2 E-46980 Paterna, Valencia, Spain

${ }^{i} L A M$ - Laboratoire d'Astrophysique de Marseille, Pôle de l'Étoile Site de Château-Gombert, rue Frédéric Joliot-Curie 38, 13388 Marseille Cedex 13, France

${ }^{j}$ National Center for Energy Sciences and Nuclear Techniques, B.P.1382, R. P.10001 Rabat, Morocco

${ }^{k} I N F N$ - Laboratori Nazionali del Sud (LNS), Via S. Sofia 62, 95123 Catania, Italy

${ }^{l}$ Nikhef, Science Park, Amsterdam, The Netherlands

${ }^{m}$ Huygens-Kamerlingh Onnes Laboratorium, Universiteit Leiden, The Netherlands

${ }^{n}$ Institute for Space Science, RO-077125 Bucharest, Măgurele, Romania
} 


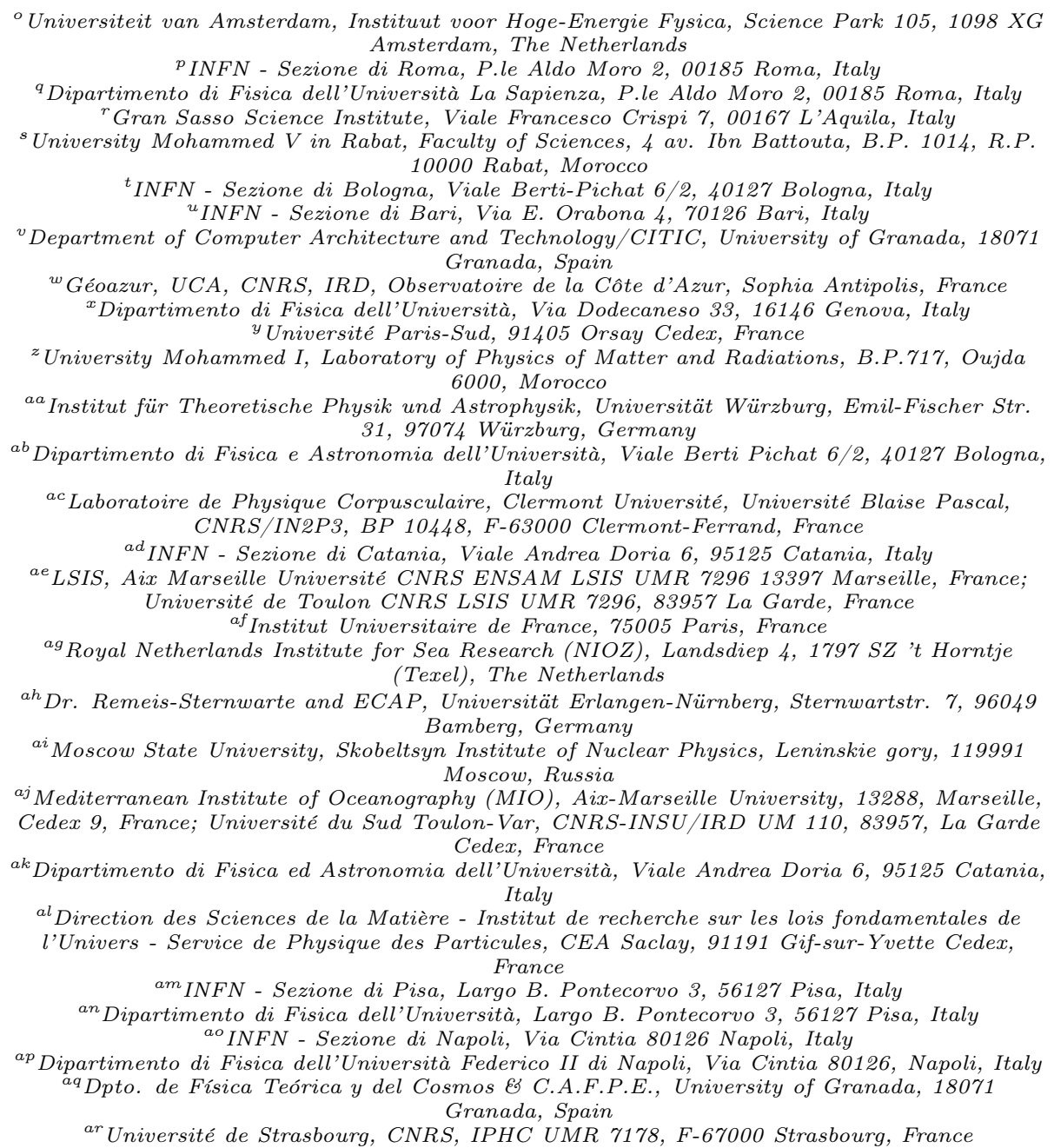

\section{Abstract}

Muons created by $\nu_{\mu}$ charged current (CC) interactions in the water surrounding the ANTARES neutrino telescope have been almost exclusively used so far in searches for cosmic neutrino sources. Due to their long range, highly energetic muons inducing Cherenkov radiation in the water are reconstructed with dedicated algorithms that allow the determination of the parent neutrino direction with a median angular resolution of about $0.4^{\circ}$ for an $E^{-2}$ neutrino spectrum. In this paper, an algorithm optimised for accurate reconstruction of energy and direction of shower events in the ANTARES detector is presented. Hadronic 
showers of electrically charged particles are produced by the disintegration of the nucleus both in $\mathrm{CC}$ and neutral current (NC) interactions of neutrinos in water. In addition, electromagnetic showers result from the CC interactions of electron neutrinos while the decay of a tau lepton produced in $\nu_{\tau} \mathrm{CC}$ interactions will in most cases lead to either a hadronic or an electromagnetic shower. A shower can be approximated as a point source of photons. With the presented method, the shower position is reconstructed with a precision of about $1 \mathrm{~m}$; the neutrino direction is reconstructed with a median angular resolution between $2^{\circ}$ and $3^{\circ}$ in the energy range of $1 \mathrm{TeV}$ to $1000 \mathrm{TeV}$. In this energy interval, the uncertainty on the reconstructed neutrino energy is about $5 \%$ to $10 \%$. The increase in the detector sensitivity due to the use of additional information from shower events in the searches for a cosmic neutrino flux is also presented.

\section{Introduction}

ANTARES [1] is the world's first deep sea neutrino telescope. The first detector elements were deployed in March 2006 and data taking started soon after. The construction was completed by mid-2008. Until recently, only muons created by muon neutrino charged current $\left(\nu_{\mu} \mathrm{CC}\right)$ interactions in the water that surrounds the detector or in the rock beneath it have been used in searches for cosmic neutrino sources. Highly energetic muons induce Cherenkov radiation in the water at a characteristic angle of $\vartheta_{\mathrm{Ch}} \approx 42^{\circ}$, which gets recorded by the detector's optical modules. The charge and timing information of the photondetections - referred to as hits - are used to reconstruct the direction of the parent neutrino with a median angular resolution of $\xi_{\text {track }} \approx 0.4^{\circ}$ for an $E^{-2}$ spectrum [2]. However, muon tracks constitute only a part of the possible event signatures of astrophysical neutrinos. Charged current interactions of electron neutrinos $\left(\nu_{e} \mathrm{CC}\right)$ create a shower of electrically charged particles. All neutrino flavours can interact through neutral current (NC). In these interactions, only a small fraction of the neutrino energy is transferred to a hadronic shower. The residual energy is carried away by the neutrino. Furthermore, tau leptons produced in $\nu_{\tau} \mathrm{CC}$ interactions decay with a branching ratio of $17 \%$ into the muon channel, $65 \%$ into a hadronic and $18 \%$ into an electromagnetic shower.

Due to neutrino oscillation, the cosmic neutrino flux measured at Earth should constitute a flavour ratio around $\Phi_{\nu_{e}}: \Phi_{\nu_{\mu}}: \Phi_{\nu_{\tau}}=1: 1: 1[3]$. Especially in the light of the recent discovery of high-energy cosmic neutrinos by the IceCube experiment, where shower events provided the majority of the neutrino candidates [4], it becomes much more important to increase the sensitivity to channels that produce particle showers. A major advantage of showers compared to muon tracks is their inherently low background: The main background for neutrino telescopes is comprised of tracks by atmospheric muons which are topologically different from showers. Misidentified muons and electron neutrinos produced by cosmic rays in the upper atmosphere present the main background in the shower channel. The rate at which electron neutrinos are produced in the 
atmosphere at the energy of interest of neutrino telescopes $(1 \mathrm{TeV}$ to $1000 \mathrm{TeV})$ is more than a factor of 10 less compared to atmospheric muon neutrinos.

High-energy muons can travel straight for several kilometres through the rock and water surrounding the detector. Showers, on the other hand, deposit all their energy within a few metres from their interaction vertex. For ANTARES they can be approximated as a point source that emits light in all directions, though with more intensity at the Cherenkov angle with respect to the direction of the parent neutrino. An early reconstruction method for showers has been already used for the search of a diffuse flux of cosmic neutrinos [5]. However, the method provided insufficient angular accuracy for point-source searches compared to the sensitivity level reached using track events.

In this paper, an algorithm optimised for accurate reconstruction of energy and direction of shower events in the ANTARES detector (section 2) is presented. The reconstruction of the shower position is described in section 3, while the directional and energy reconstruction and accuracy are presented in section 4 . The performances of the method are discussed in section 5 . The results (section 6) justify adding the selected shower events to a combined search for neutrino point-sources, as summarised in section 7 .

\section{The ANTARES detector}

The ANTARES neutrino telescope is located in the Mediterranean Sea $40 \mathrm{~km}$ off the coast of Toulon, France, at $42^{\circ} 48^{\prime} \mathrm{N}, 6^{\circ} 10^{\prime} \mathrm{E}$. The detector comprises 12 vertical lines anchored at a depth of about $2475 \mathrm{~m}$ and spaced such that for each line, the closest neighbouring line is located at a distance between about $60 \mathrm{~m}$. Each line is formed by a chain of 25 storeys with an inter-storey distance of $14.5 \mathrm{~m}$. Every storey holds 3 optical modules (OMs) housing a single $10^{\prime \prime}$ photomultiplier tube (PMT) looking downward at an angle of $45^{\circ}$. The read-out achieves relative time-stamping precision of a nanosecond between the OMs [6, 7]. At the ANTARES site, the transparency and transmission properties of the sea water [8] allow for an excellent timing measurement of the Cherenkov light induced by relativistic charged particles.

The ANTARES detector has been built in the deep-sea where all daylight is blocked. However, it is not completely dark in these depths. Seawater contains the radioactive isotope ${ }^{40} \mathrm{~K}$ which decays emitting a relativistic electron. This process produces in each of the ANTARES PMTs a continuous, ubiquitous background of around $40 \mathrm{kHz}[9]$. Additionally, microscopic life forms (mostly bacteria and plankton) are emitting their own light. This effect is called bioluminescence and contributes to the almost constant baseline rate and also occurs localised in short bursts of a few seconds. These bursts can cause count rates of several megahertz.

To estimate the reconstruction performance and develop event selection criteria, Monte Carlo simulations of the different signal and background channels are employed. Atmospheric muons are simulated using the MUPAGE package [10, 11], whereas neutrinos are simulated with the GENHEN event generator [12]. The same sample of simulated events is used for atmospheric and 
astrophysical neutrinos with an event-by-event weight to reflect the corresponding neutrino fluxes. For the atmospheric component, the flux estimate from the Bartol group is used [13]. The light propagation and the number of photons arriving on the PMTs is simulated using the KM3 programme [14, 15] and the optical background is extracted directly from the data following a run-by-run approach [16].

The longitudinal development of an electromagnetic shower is a well-understood process governed by the high-energy part of the shower. As described in section 33 of [17], the mean longitudinal profile of the energy deposition in an electromagnetic shower is reasonably well described by an analytic distribution. This function is expressed in terms of the scale variable $t=x / X_{0}$, in which the propagated distance is measured in units of radiation length $X_{0}\left(X_{0} \approx\right.$ $36 \mathrm{~g} \mathrm{~cm}^{-2}$ for water). The shape of this distribution was reproduced by our Monte Carlo simulations of electrons in water. The maximum of the shower lies between about $0.6 \mathrm{~m}$ (at $1 \mathrm{GeV}$ ) and $7 \mathrm{~m}$ (at $100 \mathrm{PeV}$ ) from the interaction vertex. Compared to the distances between the OMs in the detector, even the most energetic showers are compact enough to be approximated by a pointsource of light. Since most charged particles created in the shower propagate roughly towards the original neutrino direction, most of the photons are still emitted under the Cherenkov angle $\vartheta_{\mathrm{Ch}}$ with respect to the parent neutrino direction. This anisotropy in the number of emitted photons will be exploited to reconstruct the direction of the shower and thereby to approximate the parent neutrino direction as described in section 4 .

\section{Position reconstruction}

A proper hit selection is crucial to filter out unwanted background hits caused by the decay of ${ }^{40} \mathrm{~K}$ and bioluminescence. For the reconstruction of the shower position, the subset of hits compatible with a common source of emission, is identified. Every pair of hits $i, j$ has to fulfil the following causality criterion:

$$
\left|\vec{r}_{i}-\vec{r}_{j}\right| \geq \mathrm{c}_{\mathrm{w}} \cdot\left|t_{i}-t_{j}\right|
$$

with:

$\vec{r}_{i}$, the position of the PMT that recorded hit $i$,

$t_{i}$, the time at which hit $i$ was recorded and

$\mathrm{c}_{\mathrm{w}}$, the speed of light in water.

To understand equation (1), imagine the position $\vec{r}_{\text {shower }}$ exactly between two PMTs $i$ and $j$. Their $\Delta r=\left|\vec{r}_{i}-\vec{r}_{j}\right|$ can be arbitrarily high but $\Delta t=\left|t_{i}-t_{j}\right|$ is exactly zero. For a generic position $\vec{r}_{\text {shower }}$ and two PMTs close together $(\Delta r$ about 0 ), they have to record their hits at the same time, and thus $\mathrm{c}_{\mathrm{w}} \cdot \Delta t$ must be small as well. Thus, the time difference between two neighbouring PMTs cannot be arbitrarily high if they see the same shower, but the time difference between two arbitrarily distant hits can be zero. This procedure typically selects between 30 and 60 hits for $\nu_{e} \mathrm{CC}$ interactions from cosmic neutrinos following an 
$E^{-2}$ spectrum. Without this hit selection, one would additionally expect about one hit per OM from the ambient background. Under the above condition, this common origin of emission - i.e. the shower position $\vec{r}_{\text {shower }}$ and time $t_{\text {shower }}-$ can be determined assuming the following system of quadratic equations:

$$
\left(\vec{r}_{i}-\vec{r}_{\text {shower }}\right)^{2}=\mathrm{c}_{\mathrm{w}}^{2} \cdot\left(t_{i}-t_{\text {shower }}\right)^{2},
$$

with $1 \leq i \leq N$, where $N$ is the number of selected hits. The system of equations is linearised by taking the difference between every pair of equations $i$ and $j$ :

$$
\left(\overrightarrow{r_{i}}-\vec{r}_{j}\right) \cdot \vec{r}_{\text {shower }}-\left(t_{i}-t_{j}\right) \cdot t_{\text {shower }} c_{\mathrm{w}}^{2}=\frac{1}{2}\left[\left|\vec{r}_{i}\right|^{2}-\left|\vec{r}_{j}\right|^{2}-\mathrm{c}_{\mathrm{w}}^{2}\left(t_{i}^{2}-t_{j}^{2}\right)\right]
$$

for all $i, j: 1 \leq i<j \leq N$. The resulting system of linear equations can be written as:

$$
\mathbf{A} \vec{v}=\vec{b}
$$

with:

$\vec{v}=\left(\vec{r}_{\text {shower }}, t_{\text {shower }}\right)$, the four-dimensional space-time vector of the shower position,

$$
\begin{gathered}
\mathbf{A}=\left(\begin{array}{cccc}
\left(x_{1}-x_{2}\right) & \left(y_{1}-y_{2}\right) & \left(z_{1}-z_{2}\right) & -\left(t_{1}-t_{2}\right) \mathrm{c}_{\mathrm{w}} \\
\vdots & \vdots & \vdots & \vdots \\
\left(x_{i}-x_{j}\right) & \left(y_{i}-y_{j}\right) & \left(z_{i}-z_{j}\right) & -\left(t_{i}-t_{j}\right) \mathrm{c}_{\mathrm{w}} \\
\vdots & \vdots & \vdots & \vdots \\
\left(x_{N-1}-x_{N}\right) & \left(y_{N-1}-y_{N}\right) & \left(z_{N-1}-z_{N}\right) & -\left(t_{N-1}-t_{N}\right) \mathrm{c}_{\mathrm{w}}
\end{array}\right), \\
\vec{b}=\frac{1}{2} \cdot\left(\begin{array}{c}
\left|\vec{r}_{1}\right|^{2}-\left|\vec{r}_{2}\right|^{2}-\mathrm{c}_{\mathrm{w}}^{2}\left(t_{1}^{2}-t_{2}^{2}\right) \\
\vdots \\
\left|\vec{r}_{i}\right|^{2}-\left|\vec{r}_{j}\right|^{2}-\mathrm{c}_{\mathrm{w}}^{2}\left(t_{i}^{2}-t_{j}^{2}\right) \\
\vdots \\
\left|\vec{r}_{N-1}\right|^{2}-\left|\vec{r}_{N}\right|^{2}-\mathrm{c}_{\mathrm{w}}^{2}\left(t_{N-1}^{2}-t_{N}^{2}\right)
\end{array}\right)
\end{gathered}
$$

The matrix $\mathbf{A}$ has $M=N \cdot(N-1) / 2$ rows, therefore equation (4) represents an over-constrained system of $M$ equations that can be solved by the method of linear least square fit:

$$
\vec{v}_{\text {l.s. }}=\left(\mathbf{A}^{\mathrm{T}} \mathbf{A}\right)^{-1} \mathbf{A}^{\mathrm{T}} \vec{b}
$$

A subsequent fit is performed using a robust estimator with the previous fit as starting point and minimising the so called $M$-estimator, a modified $\chi^{2}$-like quantity, defined as:

$$
M_{\mathrm{Est}}=\sum_{i=1}^{N}\left(q_{i} \cdot \sqrt{1+t_{\mathrm{res} i}^{2} / 2}\right),
$$


with $q_{i}$, the charge of hit $i$ and

$$
t_{\text {res } i}=t_{i}-t_{\text {shower }}-\left|\vec{r}_{i}-\vec{r}_{\text {shower }}\right| / c_{\mathrm{w}},
$$

the time residual of hit $i$.

Like the $\chi^{2}$ function, $M_{\text {Est }}$ behaves quadratically for small values of $t_{\text {res }}$ but becomes asymptotically linear for larger values. Consequently, it is less sensitive to outliers, e.g. hits from ambient background or scattered photons which do not fulfil the strict relation in equation (2). The minimisation is performed by the TMinuit2 class within the ROOT framework [18.

\section{Direction and energy reconstruction}

The procedure that determines the shower direction (direction fit) makes use of a second dedicated hit selection performed on the full set of hits in the event. In particular, the charges of all hits on a given PMT in a time residual window of $-200<t_{\text {res }} / \mathrm{ns}<500$ with respect to the already performed position fit are summed up to yield $q_{i}$.

A likelihood function is defined to describe the probability $P\left(q_{i}\right)$ that a hypothetical neutrino $\nu$ with energy $E_{\nu}$, direction $\vec{p}_{\nu}$ and creating a shower at position $\vec{r}_{\text {shower }}$ causes hits with a total measured charge $q_{i}$ on a PMT $i$. The measured charge is compared to the expectation value of the number of photons on this PMT for such a shower. This expectation value depends on the neutrino energy $E_{\nu}$, the distance $d_{i}$ of the OM to the nominal shower position, the photon emission angle $\phi_{i}$ from the neutrino direction and its incident angle $\alpha_{i}$ on the PMT photocathode1. A schematic overview of the geometric variables that enter this signal part of the likelihood function is given in figure 1 . The likelihood also takes into consideration that the hit could be caused by ambient background and evaluates the probability that a background event causes a charge as observed on the PMT $\left(P_{\mathrm{bg}}\left(q_{i}\right)\right)$. The PMTs that did not record any hits which passed the hit selection are also taken into account $\left(P\left(q_{i}=0\right)\right)$.

The likelihood is given by:

$$
\begin{aligned}
\mathscr{L}= & \sum_{i=1}^{N} \log \left\{P\left(q_{i} \mid E_{\nu}, d_{i}, \phi_{i}, \alpha_{i}\right)+P_{\mathrm{bg}}\left(q_{i}\right)\right\} \\
& +\sum_{i=1}^{N^{\prime}} \log \left\{P\left(q_{i}=0 \mid E_{\nu}, d_{i}, \phi_{i}, \alpha_{i}\right)\right\},
\end{aligned}
$$

with $N$, the number of PMTs with hits, $N^{\prime}$, the number of PMTs with no hits.

\footnotetext{
${ }^{1}$ Note that in case of scattering, a Cherenkov photon does not travel along the shortest connection between the shower position and the OM, which defines the distance $d_{i}$ (cf. figure 11. The angles $\phi_{i}$ and $\alpha_{i}$ are defined w.r.t. the shortest connection, irrespective of the actual path of a scattered photon.
} 


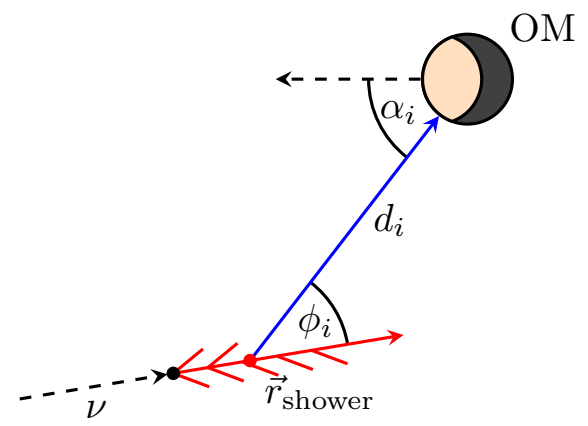

Figure 1: Geometric variables considered by the likelihood function in equation (8): photon emission angle $\phi_{i}$, showerOM distance $d_{i}$ and photon incident angle $\alpha_{i}$ on the PMT photocathode $^{1}$.

\subsection{The signal term $-P\left(q_{i} \mid E_{\nu}, d_{i}, \phi_{i}, \alpha_{i}\right)$}

The signal term of the likelihood function is determined from a three-dimensional table obtained from Monte Carlo simulations. It contains, for a given distance between shower and OM $d_{i}$, photon-emission angle $\phi_{i}$ and photonimpact angle $\alpha_{i}$, the expectation value of the number of photons on this PMT for a $1 \mathrm{TeV}$ neutrino: $\mathscr{N}_{0}\left(d_{i}, \phi_{i}, \alpha_{i}\right)$. The number of emitted photons - and, therefore, the number $\mathscr{N}_{i}$ of expected photons on the PMT - is proportional to the neutrino energy. For energies different from $1 \mathrm{TeV}$, the number of photons is scaled accordingly:

$$
\mathscr{N}_{i}=\mathscr{N}\left(E_{\nu}, d_{i}, \phi_{i}, \alpha_{i}\right)=\mathscr{N}_{0}\left(d_{i}, \phi_{i}, \alpha_{i}\right) \times E_{\nu} / 1 \mathrm{TeV} .
$$

The probability to detect $n$ photons when $\mathscr{N}$ are expected is given by the Poisson distribution:

$$
P(n \mid \mathscr{N})=\frac{\mathscr{N}^{n}}{n !} \mathrm{e}^{-\mathscr{N}}
$$

To first order, the charge $\mathcal{Q}$ expected to be measured by an ideal PMT is assumed to be proportional to the number of photons $n$ detected by the PMT:

$$
\mathcal{Q}=n \times \text { pe, }
$$

with pe (or photo-electron), the average charge measured by the PMT caused by a single photon. However, this number of photons $n$ cannot be measured with absolute precision. In reality, the measured PMT charge $q$ is affected by an uncertainty in form of a Gaussian centred around the expected charge $\mathcal{Q}$ with width proportional to $\sqrt{n}$. For simplicity, this smearing of the charge is approximated by a continuous extension of the Poisson formula that uses the Gamma function $\Gamma$, defined for real numbers $q^{\prime}=q /$ pe:

$$
P\left(q^{\prime} \mid \mathscr{N}\right)=\frac{\mathscr{N}^{q^{\prime}}}{\Gamma\left(q^{\prime}-1\right)} \mathrm{e}^{-\mathscr{N}} .
$$

Moreover, the read-out electronics saturates at charges above about 20 pe preventing the proper determination of the number of arriving photons for large signals. For this reason, to obtain a reasonable probability for the measured charge, measured charges and expected charges above 20 pe are treated as being at 20 pe. 


\subsection{The non-hit term $-P\left(q_{i}=0 \mid E_{\nu}, d_{i}, \phi_{i}, \alpha_{i}\right)$}

The probability to have a non-hit PMT $i$ is simply the Poisson probability to have zero charge while expecting $\mathscr{N}_{i}$ photons to arrive on the photocathode:

$$
P\left(q_{i}=0 \mid E_{\nu}, d_{i}, \phi_{i}, \alpha_{i}\right)=P\left(q_{i}=0 \mid \mathscr{N}_{i}\right)=\mathrm{e}^{-\mathscr{N}_{i}} .
$$

\subsection{The background term $-P_{b g}\left(q_{i}\right)$}

The background term gives the probability that one of the uncorrelated background sources - explained in section 2 - causes the observed charge $q_{i}$. Figure 2 shows the unbiased distribution of the charge caused by environmental and atmospheric background. The average value for the shown distribution is $Q_{\mathrm{bg}}=1.1 \mathrm{pe}$.

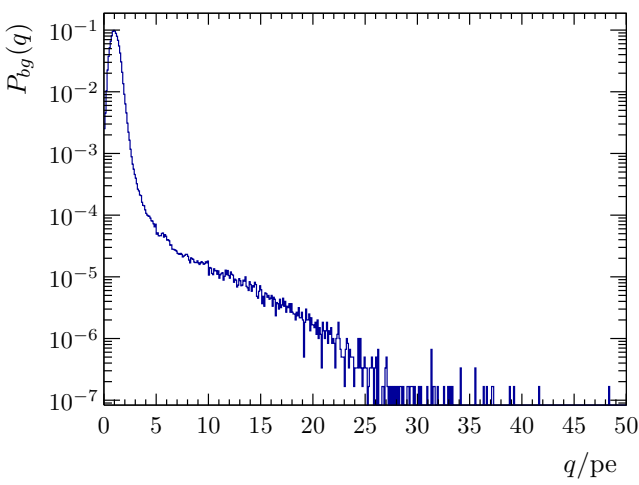

Figure 2: Unbiased distribution of the charge caused by environmental and atmospheric background.

\subsection{Implementation}

The energy and direction fit is performed with the TMinuiT2 class as well. The probability density function (PDF) used in the fit is provided as a table with discrete bins. The minimiser algorithms require the likelihood function to have well defined derivative at each point. Therefore, the PDF is interpolated with the method of trilinear interpolation. In order to find the global minimum and avoid possible local minima in the likelihood landscape, the energy-direction fit has been performed with 12 different starting directions, corresponding to the directions of the corners of an icosahedron (as seen from its centre). In the end, the fit with the maximum likelihood value is selected as the final energydirection estimate.

\subsection{Error estimator}

The direction fit also provides an angular error estimate $\beta_{\text {shower }}$ on the fit direction. After the best direction has been determined, the likelihood landscape around the fit is scanned along concentric circles of angular distances iteratively increasing in one-degree steps. The largest angular distance for which the difference between the likelihood value of any of the test directions and of the best-fit value is still smaller than 1 is used as the angular error estimate $\beta_{\text {shower }}$. 


\section{Reconstruction performance}

The performance of the reconstruction algorithm is evaluated applying it to contained events for which the simulated neutrino interaction vertex lies inside the instrumented detector volume (horizontal distance from the detector centre $\rho_{\mathrm{MC}}<90 \mathrm{~m}$ and vertical distance from the detector centre $\left.\left|z_{\mathrm{MC}}\right|<200 \mathrm{~m}\right)$. A cut on the angular error estimator was applied as well $\left(\beta_{\text {shower }}<10^{\circ}\right)$.

\subsection{Position reconstruction}

Since the reconstruction assumes one common point of emission for all photons, it will most likely reconstruct a position along the shower axis and not the actual neutrino interaction vertex. Instead, the shower position corresponds to the intensity weighted mean position of the light emission spectrum for electromagnetic showers as parametrised in reference [17]. Figure 3 shows the longitudinal and perpendicular offset of the shower position fit with respect to the simulated neutrino interaction vertex. For $\nu_{e} \mathrm{CC}$ and $\mathrm{NC}$ induced showers, the reconstructed position along the shower axis agrees well with the expected offset from the electromagnetic shower parametrisation. The median perpendicular distance to the neutrino axis is of the order of half a metre for both charged and neutral current events over a wide energy range.
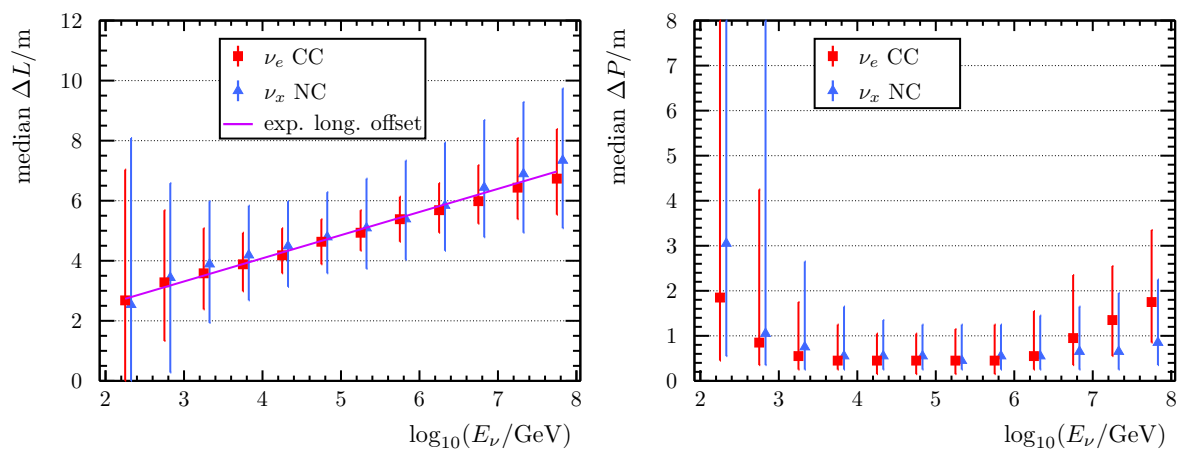

Figure 3: Distance between the true position of the neutrino interaction vertex and the reconstructed shower position in the longitudinal (left) and perpendicular (right) directions along the neutrino axis. The markers correspond to electromagnetic (red) and hadronic (blue) showers after applying the containment and angular error cuts $\left(\rho_{\mathrm{MC}}<90 \mathrm{~m}\right.$, $\left.\left|z_{\mathrm{MC}}\right|<200 \mathrm{~m}, \beta_{\text {shower }}<10^{\circ}\right)$. The purple line indicates the expected longitudinal offset from the neutrino interaction vertex for electromagnetic showers. The error bars show the $68 \%$ spread of the distribution in each energy bin.

\subsection{Direction reconstruction}

The shower angular resolution is defined as the median angle $\xi_{\text {shower }}$ between the simulated neutrino and the reconstructed shower directions. As shown in 
figure 4 (left), for contained events and energies in the range $1 \lesssim E_{\nu} / \mathrm{TeV} \lesssim 10^{3}$ it reaches values as low as $2.3^{\circ}$ with $16 \%$ of the events below $1^{\circ}$. For neutrino energies below $1 \mathrm{TeV}$, there is not enough light produced to illuminate a sufficient number of PMTs for a proper reconstruction. Above $E_{\nu} \approx 10^{3} \mathrm{TeV}$, the read-out electronics is starting to saturate and the limited size of the ANTARES detector prevents accessing higher energies with proper resolutions.

Since only a small fraction of the neutrino energy is transferred to the nucleus in NC interactions, a hadronic shower created by a high-energy neutrino has correspondingly less energy than an electromagnetic shower created by an electron neutrino of the same energy in a CC interaction. For this reason, the angular resolution for hadronic showers above $10^{3} \mathrm{TeV}$ does not deteriorate as quickly with increasing neutrino energy as for electromagnetic showers.
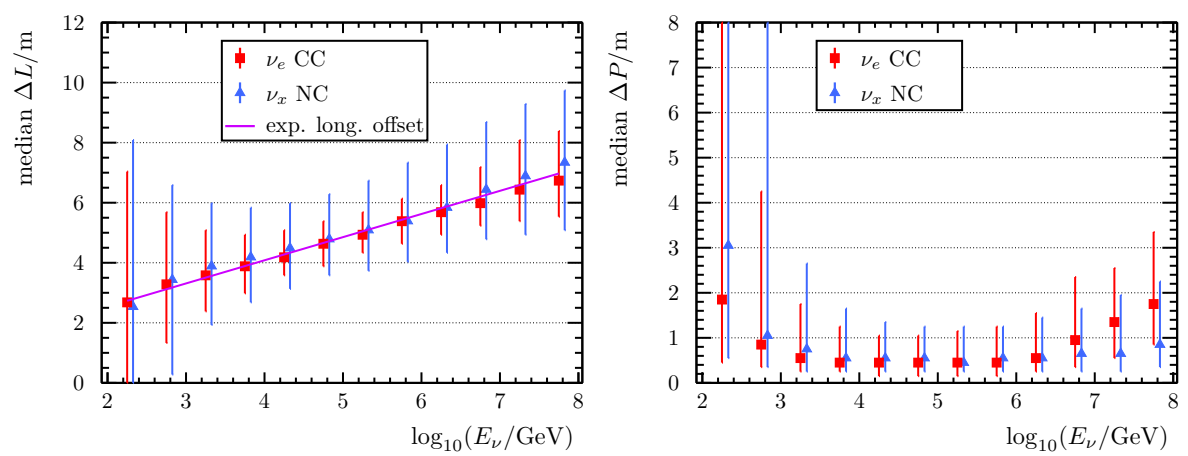

Figure 4: Left: Median angle between the directions of the reconstructed shower and the Monte Carlo neutrino as a function of the neutrino energy. Right: Median ratio of the reconstructed energy and the Monte Carlo shower energy as a function of the Monte Carlo shower energy, i.e. the sum of the energy of all mesons and charged leptons produced in the initial neutrino interaction. The markers show electromagnetic (red) and hadronic (blue) showers after the containment and angular error cuts $\left(\rho_{\mathrm{MC}}<90 \mathrm{~m}\right.$, $\left.\left|z_{\mathrm{MC}}\right|<200 \mathrm{~m}, \beta_{\text {shower }}<10^{\circ}\right)$. The error bars show the $68 \%$ spread of the distribution in each energy bin.

\subsection{Energy}

As shown in figure 4 (right), a statistical resolution of the shower energy (which is equal to the neutrino energy only for $\nu_{e} \mathrm{CC}$ events) of $5 \%$ to $10 \%$ has been achieved. A systematic underestimation of about $20 \%$ in the reconstructed energy can be observed over the whole energy range. This effect is corrected by unfolding the reconstructed energy with the right plot of figure 4 so that the median ratio between reconstructed and true Monte Carlo shower energy is flat at 1 (see figure 5). This energy correction focuses entirely on $\nu_{e} \mathrm{CC}$ events and does not produce a reliable energy estimate for the neutrino energy in NC events. The systematic effect of the energy estimation on a combined set 
comprising $\mathrm{NC}$ and $\nu_{e} \mathrm{CC}$ events can be accounted for in the specific analyses using this method.

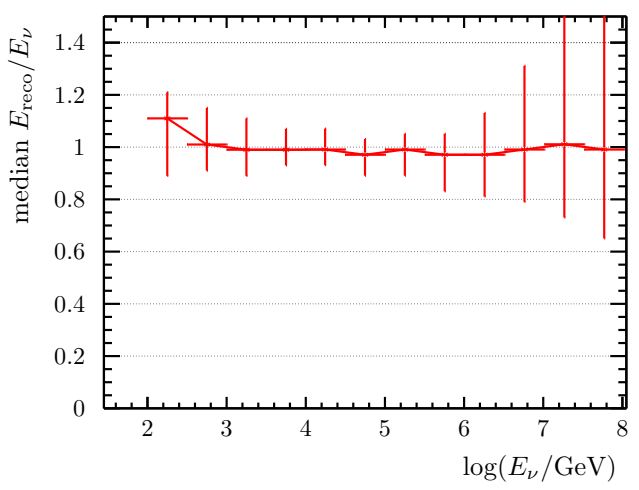

Figure 5: Ratio between reconstructed energy and $\mathrm{MC}$ shower energy for $\nu_{e} \mathrm{CC}$ events corrected for the bias seen in figure 4 The performance is shown for $\nu_{e} \mathrm{CC}$ events after the containment and angular error cuts $\left(\rho_{\mathrm{MC}}<90 \mathrm{~m}\right.$, $\left.\left|z_{\mathrm{MC}}\right|<200 \mathrm{~m}, \beta_{\text {shower }}<10^{\circ}\right)$. The error bars show the $68 \%$ spread of the distribution in each energy bin.

\subsection{Angular resolution measured in data}

The angular resolution of the shower reconstruction can also be measured directly in data using a sample of atmospheric muons. Muons can induce electromagnetic showers through stochastic energy loss processes. These muon-induced showers will have approximately the same direction as the muon. As the muon is accurately reconstructed by the track fit, a sample of electromagnetic showers of known direction can be isolated and the reconstructed shower direction compared to the direction of the reconstructed muon track. Figure 6 shows the result for a loose selection (i.e. containment, M-estimator, error estimator and GridFit ratio [19] as explained in the next section and shown in table 11). A clear population of well reconstructed showers is visible, with a resolution of two to three degrees (maximum of the distribution). This peak is well modelled in simulations of atmospheric muons, which implies that the Monte Carlo can be reliably used to determine the resolution for showers of cosmic origin. A cut of $5^{\circ}$ on the angle between the directions of the simulated and the reconstructed muon has been applied to ensure that the peak is populated with truly well-reconstructed events.

\section{Event selection and data - Monte Carlo comparison}

The discrimination of the showers produced by astrophysical neutrinos from the showers produced by the background of atmospheric muons and neutrinos is a challenging task. The main limitation is the worse angular resolution compared to muon tracks [2] and the fact that muons can also induce electromagnetic showers along their track. The advantage is the much better energy resolution.

In the following, the performances of the algorithm to discriminate cosmic showers from the atmospheric background using Monte Carlo observable variables is described. The cosmic signal is characterised by a power law function 


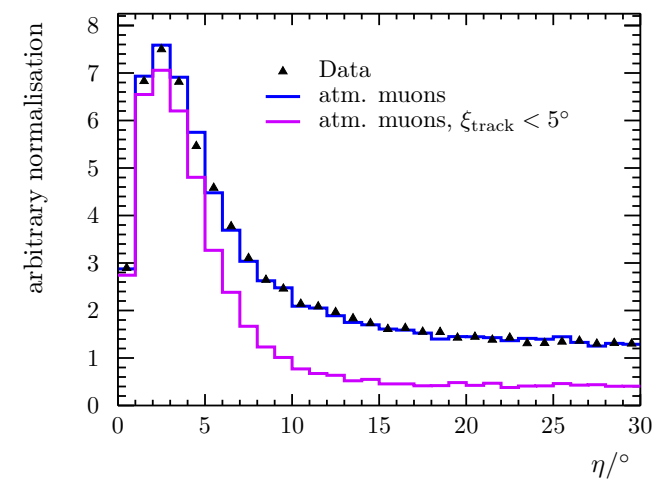

Figure 6: The angular separation $\eta$ of the reconstructed directions using track and shower hypotheses applied to the same atmospheric muon events. The angle is shown for data (black), simulated atmospheric muons (blue) and simulated atmospheric muons reconstructed with an angular error less than $5^{\circ}$ (purple).

of the energy with a harder spectral index. The results obtained in this section are only illustrative of the methods and can be adapted according to the specific requirements of different analyses. In particular, the results of a first all-flavour neutrino point-like source search using nine years of the ANTARES data are already public [20]. The focus in the following is mainly on the reduction of the atmospheric muon contamination, while maintaining the largest fraction of the cosmic signal. In particular, the exact cut values have not been optimised (e.g. for best sensitivity or discovery potential in the point-source search).

The effect of the cuts on different Monte Carlo samples are presented in table 1. The first two columns indicate the name and the value of the applied condition. Two of them are already presented: the "up-going" condition requires that showers are reconstructed with $\cos \left(\vartheta_{\text {shower }}\right)>-0.1$; the "error estimate" requires that the angular error estimate is $\beta_{\text {shower }}<10^{\circ}$. The other criteria are described in the following. The effect on the atmospheric muon sample is presented in column $3\left(\epsilon_{\mu}^{\mathrm{atm}}\right)$; that on the atmospheric neutrinos (either yielding a shower or a muon) in column $4\left(\epsilon_{\nu \rightarrow \text { any }}^{\text {atm }}\right)$. The effect on a flux of cosmic neutrinos with spectrum $E^{-2}$ yielding showers of muons simulating a shower are presented in column $5\left(\epsilon_{\nu \rightarrow \text { shower }}^{E^{-2}}\right)$ and $6\left(\epsilon_{\nu \rightarrow \mu}^{E^{-2}}\right)$, respectively. The last row of the table shows the number of expected events in every channel. After applying these selection criteria to the ANTARES data set with an effective life time of 1690 days, 172 shower events remain. Below, the description of the other criteria applied to reduce the background is presented.

Containment+M-Estimator Reconstructing atmospheric muons with a shower algorithm often produces "shower positions" that lie far away from the detector boundary and have a large $M_{\text {Est }}$ value (equation (6) ). A rough selection on position and reconstruction quality reduces the amount of background by $70 \%$ already before the direction fit. The quantity $\rho_{\text {shower }}$ is the horizontal distance of the reconstructed shower position from the detector's centre and $z_{\text {shower }}$ is the vertical height above the detector's centre.

Track Veto To avoid an overlap between the track and shower samples, events 
that pass the muon track selection are excluded from the shower channel.

GridFit Ratio The GridFit algorithm was developed for another, recent analysis [19]. It is used here to suppress down-going muon events. In a first step, it segments the full solid angle in 500 directions. For each direction, the number of hits compatible with a muon track from this direction is determined. The GridFit ratio $R_{\mathrm{GF}}$ is the ratio between the sum of the compatible hits $N_{\mathrm{GFR}}$ for all up-going and all down-going test directions: $R_{\mathrm{GF}}=\frac{\sum_{\mathrm{up}} N_{\mathrm{GFR}}}{\sum_{\mathrm{down}} N_{\mathrm{GFR}}}$. A lower value, therefore, means a higher likelihood of this event to be a down-going muon. A selection criterion combining the GridFit ratio and the number of selected shower hits (see figure 7) was devised to further suppress the atmospheric muon background.

Likelihood Muon Veto In order to improve the discrimination between cosmic showers and atmospheric muons, a dedicated likelihood function has been developed. This likelihood considers only hits that coincide with another hit on the same storey within $20 \mathrm{~ns}$ and its PDF is based on the following parameters:

- time residual $t_{\text {res }}$ (equation 7) of the hits w.r.t. the reconstructed shower position,

- number $N$ of on-time hits $\left(-20<t_{\text {res }} / \mathrm{ns}<60\right)$ and

- distance $d$ of the hits to the reconstructed shower position.

The Likelihood is given by the following equation:

$$
\mathscr{L}_{\mu \text { Veto }}=\sum_{\text {hits }}\left[\log \left\{P_{\text {shower }} / P_{\text {muon }}\right\}+P_{\text {shower }}-P_{\text {muon }}\right],
$$

with $P_{\text {shower }}=P\left(N, d, t_{\text {res }} \mid\right.$ shower $)$ and $P_{\text {muon }}=P\left(N, d, t_{\text {res }} \mid\right.$ muon $)$. These PDFs are based on the same Monte Carlo simulations mentioned in section 2 with an energy spectrum proportional to $E^{-2}$ for the cosmic neutrinos that induce the showers. The likelihood function shown in equation (14) was developed to achieve an optimal separation of the shower and muon distributions. This likelihood parameter can be combined with the zenith angle, reconstructed by the established muon-track fitting algorithm [2]: On events that have been reconstructed as down-going a harder likelihood ratio cut can be applied. The distribution for this quantity plotted before and after the combined cut is shown in figure 8 . This method further reduces the number of atmospheric muons by more than one order of magnitude. Even so, the majority of the remaining events consists still of misreconstructed atmospheric muons.

Charge Ratio When the shower fit reconstructs a position along the muon track, one would expect photons induced by the muon to also arrive earlier than predicted by a point source hypothesis. Thus, the charge ratio between the "early" and "on-time" hits was investigated. The distribution of 

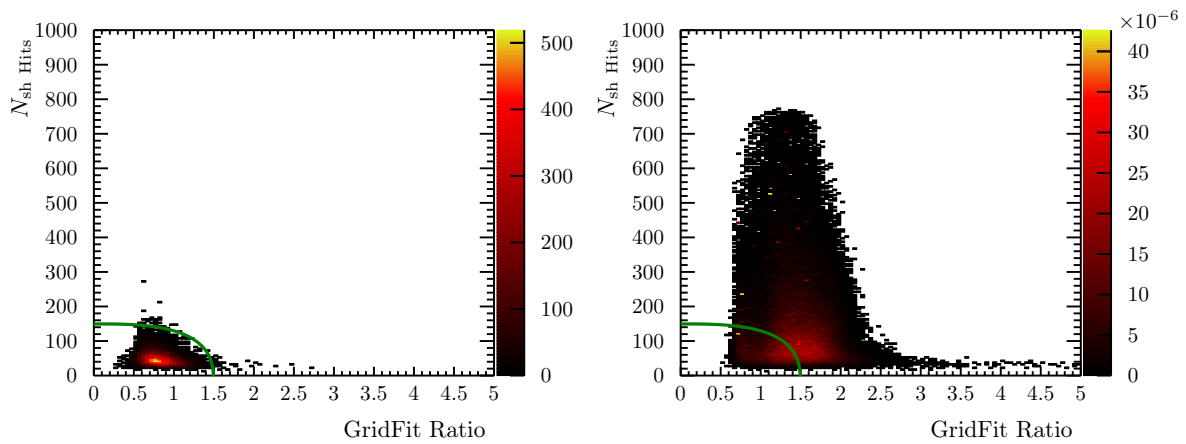

Figure 7: Distribution (colour scale on the right for the number of events) of events with number of selected hits $N_{\text {sh hits }}$ versus the GridFit ratio $R_{\text {GT }}$ (see text). The distributions are shown for atmospheric muons (left) and for cosmic electron neutrinos undergoing charged current interaction creating showers (right) after all previous cuts listed in table 1] The green line shows the combined $R_{\mathrm{GF}}-N_{\mathrm{sh} \text { hits }}$ cut: Events below the line are rejected.

the ratio of those two charge-sums is shown in figure 9. Here, $Q_{\text {early }}$ is the summed charge of all hits with a time residual of $-1000 \leq t_{\text {res }} / \mathrm{ns} \leq-40$ with respect to the reconstructed shower and $Q_{\text {on-time }}$ is the summed charge of all hits with time residuals of $-30 \leq t_{\text {res }} / \mathrm{ns} \leq 1000$.

After reducing the amount of atmospheric muons by six orders of magnitude, just before the charge-ratio cut (see figure 9), the Monte Carlo simulations of atmospheric muons do no longer well describe the data in the right part of the plot. The discrepancy lies well out of the acceptance region wherein the data agrees with the simulation of atmospheric neutrino events.

The event selection does not only reject unwanted background events but also poorly reconstructed signal events. The direction resolution improves slightly compared to what is shown in figure 4, particularly in the lower energy region.

\section{Conclusion}

An algorithm to reconstruct underwater particle showers has been developed. It makes use of the fact that the highly energetic, electrically charged particles induce Cherenkov radiation mainly under one specific angle with respect to the direction of the parent neutrino and that this emission profile is preserved over large distances due to the good optical properties of the deep-sea water. The showers can be approximated as point sources of photons which emit their light at one given time. The shower position is reconstructed with a precision of about $1 \mathrm{~m}$ and for the neutrino direction resolutions of $2^{\circ}$ to $3^{\circ}$ are achieved. A statistical uncertainty for the shower energy of about $5 \%$ to $10 \%$ is obtained.

Despite their much more compact event signature, the shower algorithm's angular resolution is only about a factor of five worse than that of the muon 

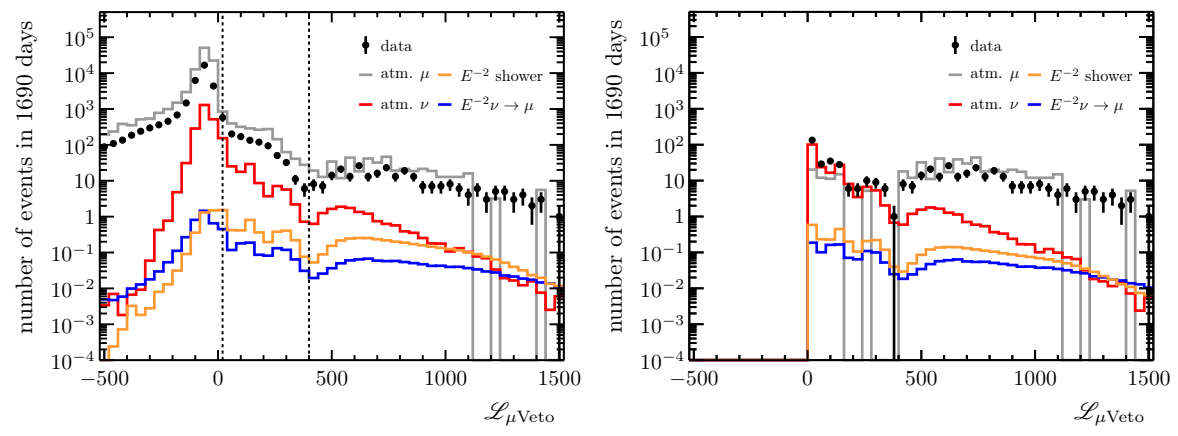

Figure 8: Likelihood muon veto distribution for atmospheric neutrinos (red), atmospheric muons (grey), showers caused by astrophysical neutrinos (orange) and data (black). The distributions are shown after the GridFit Ratio and all previous cuts listed in table 1 have been applied (left) and additionally after the likelihood-ratio cut (right). The dashed lines mark the cut values: Everything below $\mathscr{L}=20$ and everything reconstructed as $\cos \left(\vartheta_{\text {track }}\right)<-0.2$ and below $\mathscr{L}=400$ is rejected.

Table 1: Event selection criteria for the shower channel and the selection efficiency after each step for atmospheric muons and neutrinos and cosmic neutrinos creating a shower in the detector. The efficiencies are defined as the ratio of the number of events that passed a cut and the number of events after the trigger selection. In the last row, the number of events expected from the simulation is shown.

\begin{tabular}{llrrrr} 
Criterion & Condition & $\epsilon_{\mu}^{\text {atm. }}$ & $\epsilon_{\nu \rightarrow \text { any }}^{\text {atm. }}$ & $\epsilon_{\nu \rightarrow \text { shower }}^{\mathrm{E}^{-2}}$ & $\epsilon_{\nu \rightarrow \mu}^{\mathrm{E}^{-2}}$ \\
Triggered & & $100 \%$ & $100 \%$ & $100 \%$ & $100 \%$ \\
Containment & $\rho_{\text {shower }}<300 \mathrm{~m},\left|z_{\text {shower }}\right|<250 \mathrm{~m}$ & $53 \%$ & $81 \%$ & $93 \%$ & $75 \%$ \\
M-Estimator & $M_{\text {Est }}<1000$ & $40 \%$ & $66 \%$ & $90 \%$ & $72 \%$ \\
Track Veto & not selected as muon candidate & $40 \%$ & $59 \%$ & $88 \%$ & $49 \%$ \\
Up-Going & $\cos \left(\vartheta_{\text {shower }}\right)>-0.1$ & $18 \%$ & $44 \%$ & $46 \%$ & $28 \%$ \\
Error Estimate & $\beta_{\text {shower }}<10^{\circ}$ & $0.66 \%$ & $5.0 \%$ & $26 \%$ & $9.3 \%$ \\
GridFit Ratio & $\left(\frac{R_{\mathrm{G} F \mathrm{~F}}}{1.3}\right)^{3}+\left(\frac{N_{\text {sh hits }}}{150}\right)^{3}>1$ & $0.057 \%$ & $4.2 \%$ & $22 \%$ & $6.1 \%$ \\
Muon Veto & $\mathscr{L}_{\mu \text { Veto }}>\left\{\begin{array}{l}400, \quad \text { if } \cos \left(\vartheta_{\text {track }}\right)<-0.2 \\
20, \quad \text { otherwise }\end{array}\right.$ & $2.9 \times 10^{-4} \%$ & $0.41 \%$ & $17 \%$ & $2.8 \%$ \\
Charge Ratio & $\log \left(Q_{\text {early }} / Q_{\text {on-time }}\right)<-1.3$ & $1.1 \times 10^{-5} \%$ & $0.31 \%$ & $16 \%$ & $1.3 \%$ \\
\hline expected Events in 1690 days & 18.8 & 163 & 2.78 & 0.63
\end{tabular}

channel. Combined with their inherently low background, shower events will prove very valuable in the search for point-like and extended neutrino sources. Our studies [20] showed that the shower channel contributes about $23 \%$ of all signal events for an $E^{-2}$ energy spectrum corresponding to an increase of the point-source sensitivity of about $10 \%$. The sensitivity of the shower channel to the ANTARES searches for a diffuse flux of cosmic neutrinos is almost equivalent to that of the muon channel [5, 21], due to the better energy estimate and the 


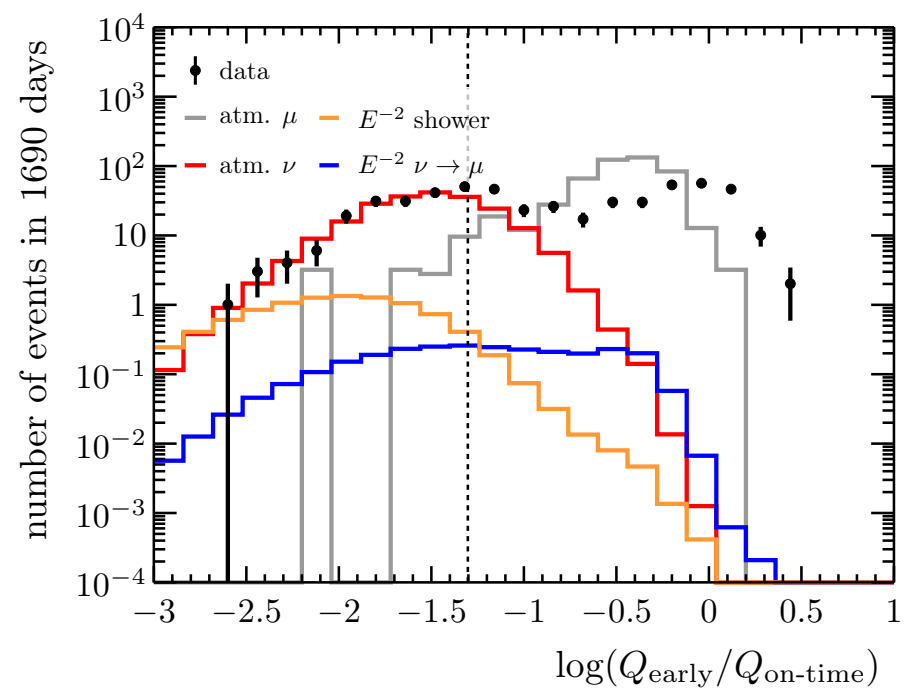

Figure 9: Distribution of the ratio of the sum of the charges for early and on-time hits for atmospheric neutrinos (red), atmospheric muons (grey), showers caused by astrophysical neutrinos (orange) and data (black). After the muon veto and all previous cuts listed in table1. The dashed line marks the cut value: Everything to the right is rejected.

lower atmospheric background.

\section{Acknowledgements}

The authors acknowledge the financial support of the funding agencies: Centre National de la Recherche Scientifique (CNRS), Commissariat à l'énergie atomique et aux énergies alternatives (CEA), Commission Européenne (FEDER fund and Marie Curie Program), Institut Universitaire de France (IUF), IdEx program and UnivEarthS Labex program at Sorbonne Paris Cité (ANR-10LABX-0023 and ANR-11-IDEX-0005-02), Labex OCEVU (ANR-11-LABX-0060) and the A*MIDEX project (ANR-11-IDEX-0001-02), Région Île-de-France (DIMACAV), Région Alsace (contrat CPER), Région Provence-Alpes-Côte d'Azur, Département du Var and Ville de La Seyne-sur-Mer, France; Bundesministerium für Bildung und Forschung (BMBF), Germany; Istituto Nazionale di Fisica Nucleare (INFN), Italy; Stichting voor Fundamenteel Onderzoek der Materie (FOM), Nederlandse organisatie voor Wetenschappelijk Onderzoek (NWO), the Netherlands; Council of the President of the Russian Federation for young scientists and leading scientific schools supporting grants, Russia; National Authority for Scientific Research (ANCS), Romania; Ministerio de Economía y Competitividad (MINECO): Plan Estatal de Investigación (refs. FPA2015-65150-C3-1P, -2-P and -3-P, (MINECO/FEDER)), Severo Ochoa Centre of Excellence and 
MultiDark Consolider (MINECO), and Prometeo and Grisolía programs (Generalitat Valenciana), Spain; Ministry of Higher Education, Scientific Research and Professional Training, Morocco. We also acknowledge the technical support of Ifremer, AIM and Foselev Marine for the sea operation and the CC-IN2P3 for the computing facilities.

\section{Bibliography}

\section{References}

[1] The ANTARES Collaboration. ANTARES: the first undersea neutrino telescope. Nuclear Instruments and Methods, A656:11, 2011.

[2] The ANTARES Collaboration. Searches for Point-like and extended neutrino sources. The Astrophysical Journal Letters, 786:L5, 2014.

[3] J. G. Learned and S. Pakvasa. Detecting $\nu_{\tau}$ oscillations at PeV energies Astroparticle Physics, 3, 1995.

[4] The IceCube Collaboration. Atmospheric and astrophysical neutrinos above $1 \mathrm{TeV}$ interacting in IceCube. Physical Review D, 91:022001, 2015.

[5] The ANTARES Collaboration. An algorithm for the reconstruction of high-energy neutrino-induced particle showers and its application to the ANTARES neutrino telescope. The European Physical Journal C, $77(6): 419,2017$.

[6] The ANTARES Collaboration. Performance of the front-end electronics of the ANTARES neutrino telescope. Nuclear Instruments and Methods A, 622:59, 2010.

[7] The ANTARES Collaboration. Time calibration with atmospheric muon tracks in ANTARES. Astroparticle Physics, 78:43, 2016.

[8] The ANTARES Collaboration. Transmission of light in deep sea water at the site of the Antares neutrino telescope. Astroparticle Physics, 23:131, 2005 .

[9] C. Tambirini, M. Canals, X. Durrieu de Madron, L. Houpert, D. Lefèvre et al. Deep-Sea Bioluminescence Blooms after Dense Water Formation at the Ocean Surface. PLoS ONE, 8(7): e67523, 2013.

[10] Y. Becherini et al. A parametrisation of single and multiple muons in the deep water or ice. Astroparticle Physics, 25, 2006.

[11] G. Carminati, A. Margiotta, and M. Spurio. Atmospheric MUons from PArametric formulas: A Fast GEnerator for neutrino telescopes (MUPAGE). Computer Physics Communications, 179, 2008. 
[12] D. Bailey. Monte Carlo tools and analysis methods for understanding the ANTARES experiment and predicting its sensitivity to Dark Matter. $\mathrm{PhD}$ thesis, Wolfson College, Oxford, 2002.

[13] V. Agrawal, T. K. Gaisser, P. Lipari and T. Stanev. Atmospheric neutrino flux above $1 \mathrm{GeV}$. Physical Review D, 53, 1996.

[14] J. Brunner. ANTARES simulation tools. Proceedings of the VLVnT, 2003. http://www.vlvnt.nl/proceedings.pdf.

[15] A. Margiotta. Common simulation tools for large volume neutrino detectors. Nuclear Instruments and Methods, 725, 2013.

[16] L. A. Fusco and A. Margiotta. The run-by-run monte carlo simulation for the ANTARES experiment. EPJ Web Conference, 116, 2016.

[17] C. Patrignani et al. [Particle Data Group]. 2015 Review of Particle Physics - Passage of particles through matter. Chinese Physics C, 40, 100001, 2016.

[18] The ROOT Data Analysis Framework. www.root.cern.ch

[19] E. L. Visser. Neutrinos from the Milky Way. PhD thesis, Nikhef, 2015.

[20] The ANTARES Collaboration. First all-flavour Neutrino Point-like Source Search with the ANTARES Neutrino Telescope. arXiv:1706.01857.

[21] The ANTARES Collaboration. Search for a diffuse flux of cosmic neutrinos with the ANTARES telescope. Proceedings of Science - ICRC, 2017. 